\title{
Integrated Process and Molecular Design with Modelica Using Continuous-Molecular Targeting
}

\author{
Christoph U. Gertig ${ }^{1} \quad$ Dominik Tillmanns $^{1} \quad$ Johannes Schilling $^{1} \quad$ Uwe Bau $^{1}$ \\ Franz Lanzerath ${ }^{1} \quad$ Joachim Gross $^{2} \quad$ André Bardow $^{1}$ \\ ${ }^{1}$ Institute of Technical Thermodynamics, RWTH Aachen University \\ Schinkelstr. 8, 52062 Aachen, Germany \\ ${ }^{2}$ Institute of Technical Thermodynamics and Thermal Process Engineering, Stuttgart University \\ Pfaffenwaldring 9, 70569 Stuttgart, Germany \\ andre.bardow@ltt.rwth-aachen.de
}

\begin{abstract}
The performance of many chemical and energy conversion processes depends on the choice of the molecules used, e.g. as solvents or working fluids. To capture the complex relations between the properties of the molecules used and the process conditions, the selection of suitable molecules should be directly integrated into process design. Solving the resulting challenging integrated design problem is enabled by the Continous-Molecular Targeting - Computer-Aided Molecular Design (CoMT-CAMD) approach. Here, the combinatorial complexity of the molecular decisions is avoided by relaxing molecular parameters in a physically-based thermodynamic model. So far, implementations of CoMTCAMD were based on procedural programming languages. This impedes reusability and the investigation of process variants as well as the design of complex processes. In order to overcome these shortcomings, we implement the CoMT-CAMD approach based on objectoriented process modeling and thus enable the integrated process and molecular design with Modelica. The resulting approach is demonstrated for the design of a process and the working fluid for a geothermal Organic Rankine Cycle application.
\end{abstract}

Keywords: GenOpt, optimization, integrated fluid and process design, computer-aided molecular design, $P C$ SAFT

\section{Introduction}

In order to achieve high performance, chemical as well as energy conversion processes have to be tailored to the specific applications. The key to tailoring a process is often the choice of suitable molecules. Examples are the selection of solvents for absorption processes (Adjiman et al., 2014; Bardow et al., 2010; Burger et al., 2015; Papadopoulos and Linke, 2009), refrigerants for compression chillers (Roskosch and Atakan, 2015; Sahinidis et al., 2003) and working fluids for Organic Rankine
Cycles (Linke et al., 2015; Bao and Zhao, 2013; Lampe et al., 2015).

Today, design methods usually separate the choice of suitable molecules and the process design (for a literature review, see e.g. Linke et al., 2015): in a first step, molecular candidates are pre-selected using criteria based on heuristics. In a second step, the pre-selected molecules are used for process optimization.

However, these two-step approaches usually lead to suboptimal solutions. Heuristic selection criteria cannot capture the strong and complex relations between the properties of chosen molecules and the corresponding optimal process conditions. Therefore, the global optimum might already be excluded from the solution space when heuristics are applied. Consequently, the design of molecules should be directly integrated into the process design (Adjiman et al., 2014; Linke et al., 2015). The direct formulation of this integrated design problem leads to a mixed integer nonlinear program (MINLP) (Gani, 2004) where each molecule considered adds one degree of freedom. Due to the large number of potential candidate molecules, the solution of this MINLP is usually prohibitively difficult.

Thus, systematic approaches have been proposed for the approximate solution of the integrated design problem: Pereira et al. $(2008 ; 2011)$ solve the integrated design problem based on property predictions with the statistical associating fluid theory for potentials of variable attractive range (SAFT-VR) with a search space limited to linear alkanes. For Organic Rankine Cycles, the review by Linke et al. (2015) summarizes the state of the art. Recently, Burger et al. (2015) have solved the integrated design problem utilizing a hierarchical approach and short-cut models for the process. Gopinath et al. (2016) have proposed an approach for the integrated design utilizing physical domain reduction. They employ tests to remove regions from the molecular and process domains where constraints, e.g., on phase behavior, are violated. 
Bardow et al. (2010) proposed a targeting-based design approach called Continuous-Molecular Targeting Computer-Aided Molecular Design (CoMT-CAMD) for the integrated molecular and process design. Here, the molecular properties are modeled by the PerturbedChain Statistical Associating Fluid Theory (PC-SAFT) equation of state (Gross and Sadowski, 2001). In PCSAFT, each fluid is described by a set of physicallybased pure component parameters. In the first step of CoMT-CAMD, the so-called Continuous-Molecular Targeting (CoMT), the discrete PC-SAFT pure component parameters are regarded as continuous degrees of freedom of the design problem and are optimized simultaneously with the process conditions (Lampe et al., 2014; Stavrou et al., 2014). The resulting design problem can be formulated as a nonlinear program (NLP) optimization problem. The results of this optimization are the set of optimal pure component parameters for a hypothetical target molecule and the corresponding optimal process parameters. In a second step, ComputerAided Molecular Design (CAMD) methods can be used to design the real molecule which best matches the optimal process performance (Lampe et al., 2015). The CoMT-CAMD approach has been applied successfully to the design of solvents for $\mathrm{CO}_{2}$ capture (Stavrou et al., 2014) and working fluids for Organic Rankine Cycles (Lampe et al., 2014; 2015). A similar targeting approach for integrated design was presented by Roskosch and Atakan (2015). They use a cubic equation of state for property modeling and relax its parameters in a simultaneous optimization of a compression heat pump process and working fluid. Subsequently, they select suitable fluids from databanks utilizing a fitted function for COP estimation.

So far, integrated process and molecular design with CoMT-CAMD was based on process models implemented in a procedural programming language. This hinders the reusability of models and complicates the design of complex processes as well as the investigation of process variants. Furthermore, the use of procedural languages is not convenient in case dynamic processes have to be investigated.

These shortcomings can be overcome by using a language suited for object-oriented and equation-based modeling like Modelica (Fritzson, 1998) to model the process. Thus, in this work, we present the first implementation of the CoMT-CAMD approach based on Modelica process models. Thereby, we enable the integrated process and molecular design with Modelica. In order to illustrate the design approach, a case study is presented for the design of a geothermal Organic Rankine Cycle (ORC) application.

The paper is structured as follows: in Section 2, the CoMT-CAMD approach is explained. In Section 3, the implementation of CoMT-CAMD based on Modelica models is described. The case study of the ORC application is presented in Section 4 before conclusions are drawn in Section 5.

\section{The CoMT-CAMD Approach}

The Continuous-Molecular Targeting - ComputerAided Molecular Design (CoMT-CAMD) approach was introduced by Bardow et al. (2010). In CoMT-CAMD, the fluids are modeled by the Perturbed-Chain Statistical Associating Fluid Theory (PC-SAFT) equation of state (EOS). Thus, any fluid can be described by a set of PCSAFT pure component parameters. The CoMT-CAMD approach comprises two main steps as shown in Figure 1.

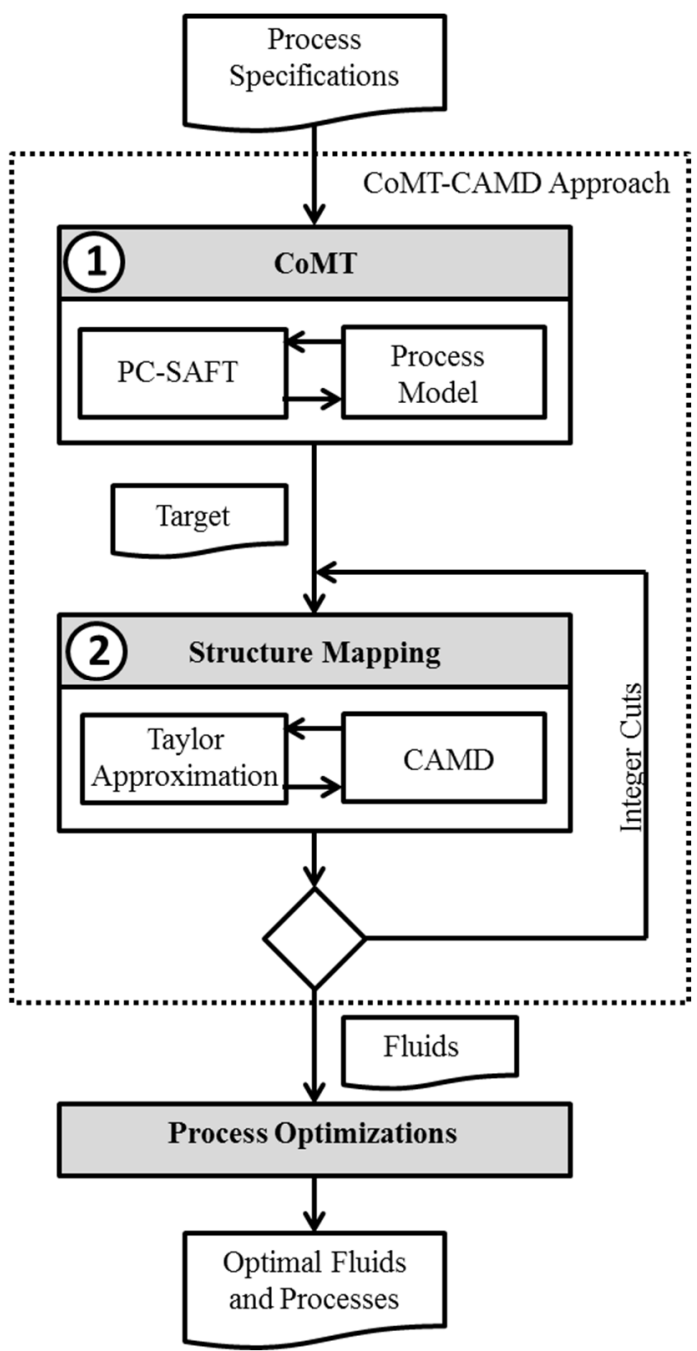

Figure 1: The workflow of the CoMT-CAMD approach.

In the first step, the so-called Continuous-Molecular Targeting (CoMT), the process conditions and the molecules are simultaneously optimized. For this purpose, the PC-SAFT pure component parameters describing the properties of the molecules are relaxed and treated as continuous variables of the optimization problem. The results of this optimization are the PC-SAFT pure component parameters of a hypothetical optimal fluid, 
the so-called target, and the corresponding optimal process conditions. In the second step, real fluids are identified in the so-called structure-mapping. For this purpose, a second-order Taylor-approximation is computed around the hypothetical optimum. This Taylor approximation is used to estimate the objective function value of processes with real fluids. In the structure-mapping, real fluids can either be selected from databanks of known fluids (Lampe et al., 2014) or designed using Computer-Aided Molecular Design (CAMD) algorithms (Lampe et al., 2015). This CAMD algorithm employs Group Contribution (GC) methods to link PCSAFT pure component parameters to molecule structures. This link is used to identify the optimal molecular structure by solving a mixed-integer quadratic program (MIQP) optimization problem (for details, see Section 2.3). Due to inaccuracies in the models and the method, usually, not only one fluid but a ranking of candidates is desired. Thus, the CAMD algorithm is applied repeatedly using integer cuts (see e.g. Fazlollahi et al., 2012) to exclude previously found molecules in each new run. The CoMT-CAMD approach thus yields a list of real fluids which best match the target. For these fluids, individual process optimizations are performed to determine the optimal process performance.

Details of the CoMT step are explained in Section 2.1 and the PC-SAFT equation of state is described in Section 2.2. The structure-mapping step of CoMT-CAMD is discussed in more detail in Section 2.3 followed by a short section on the final process optimizations.

\subsection{Continuous-Molecular Targeting}

The aim of the CoMT step is the simultaneous optimization of process conditions and fluids to obtain a target for the subsequent structure-mapping (Bardow et al., 2010). As mentioned before, the fluids are modeled with the PC-SAFT equation of state. The PC-SAFT pure component parameters used to describe the fluids are relaxed and thus treated as continuous variables of the optimization problem. This relaxation transforms the mixed-integer nonlinear program (MINLP) of the fully integrated design into a nonlinear program (NLP) given by problem (1) (Lampe et al., 2015):

$$
\begin{array}{cc}
\max _{x, y} f(x, y) & \\
\text { s.t. } g_{1}(x, y) \leq 0 \\
g_{2}(x, y)=0 \\
h(x, y)=0 & \text { "process model" } \\
\begin{array}{c}
A y \leq b \\
x_{\min } \leq x \leq x_{\max }
\end{array} & \text { "PC-SAFT" } \\
y_{\min } \leq y \leq y_{\max } & \text { "convex hull" } \\
&
\end{array}
$$

Here, $x$ is the vector with the degrees of freedom of the process and $y$ the vector containing the PC-SAFT pure component parameters of the molecules.
The objective function $f$ is, for example, a thermodynamic measure like an efficiency. The process model is formulated in terms of inequality constraints $g_{1}$ and equality constraints $g_{2}$. The PC-SAFT equation of state $h$ is used to compute thermodynamic quantities based on the pure component parameters and the process conditions. Additionally, bounds are defined on the process degrees of freedom $x_{\min }$ and $x_{\max }$ and the PC-SAFT pure component parameters $y_{\min }$ and $y_{\max }$.

Additional linear inequality constraints $(A y \leq b)$ are used which set up a convex hull around the PC-SAFT pure component parameters of real fluids (see Lampe et $a l$. (2014) for details). This ensures that the CoMT step results in a hypothetical fluid which is similar to any real substance. The NLP optimization of the CoMT step results in a hypothetical optimal fluid $y^{*}$ and optimal process conditions $x^{*}$.

In general, the pure component parameters $y^{*}$ of the optimal hypothetical fluid are not equal to those of any real fluid. Thus, real substances with favorable performance are identified in a subsequent structure-mapping (discussed in Section 2.3).

\subsection{PC-SAFT Equation of State}

The Perturbed-Chain Statistical Associating Fluid Theory (Gross and Sadowski, 2001, 2002; Gross, 2005; Gross and Vrabec, 2006) is a physically-based equation of state model for the residual Helmholtz energy. The underlying molecular picture considers molecules as chains of hard spheres (segments) which interact with each other.

Both pure fluids and fluid mixtures are described based on typically 3 to 7 parameters per each pure component. In this work, we consider only non-polar and non-associative molecules, so that 3 parameters of PCSAFT are sufficient: the segment number $m$, the segment diameter $\sigma$ and the segment dispersion energy $\varepsilon / k$.

As only the residual part of the Helmholtz energy is calculated from PC-SAFT, an additional property is required to calculate absolute caloric properties. Here, the additional property is the ideal gas heat capacity. In the CoMT step, the molecules are exclusively described by the PC-SAFT pure component parameters, which should therefore also be used in order to obtain the ideal gas heat capacity (Lampe et al., 2014). For this purpose, Quantitative Structure-Property Relationships (QSPR) are used with PC-SAFT pure component parameters as inputs to calculate ideal gas heat capacities $c^{p, i g}$ (for details see Stavrou et al., 2014; Lampe et al., 2014; 2015). Additionally, another QSPR model based on PC-SAFT pure component parameters is used to calculate molar masses (Lampe et al., 2015).

By combining the QSPR methods and PC-SAFT, all thermodynamic equilibrium properties can be calculated based on 3 PC-SAFT pure component parameters in a thermodynamically consistent form. 


\subsection{Structure-Mapping using Computer- Aided Molecular Design}

As shown in Figure 1, we use Computer-Aided Molecular Design (CAMD) in the structure-mapping step in order to design real fluids which best match the target obtained from the CoMT step. For this purpose, a measure for the expected performance of fluids in the process is needed.

A simple measure is the distance $\left\|y-y^{*}\right\|$ of a real fluid's pure component parameters from those of the hypothetical optimal fluid in the space of the PC-SAFT parameters. However, this simple measure is not appropriate since it neglects the different sensitivities of the objective function with respect to different PC-SAFT parameters and it depends on scaling. To overcome these limitations, a performance measure is calculated in the space of the objective function itself. For this purpose, we compute the following Taylor-approximation to estimate the performance:

$$
\begin{aligned}
\hat{f}(y)=f^{\mathrm{opt}}\left(y^{*}\right) & +\frac{d f^{\mathrm{opt}}}{d y}\left(y-y^{*}\right) \\
& +\frac{1}{2}\left(y-y^{*}\right)^{T} \frac{d^{2} f^{\mathrm{opt}}}{d y^{2}}\left(y-y^{*}\right) .
\end{aligned}
$$

Here, $f^{\text {opt }}$ is the objective function of Problem (1) $f(x, y)$ rewritten such that it yields the optimal performance solely based on the PC-SAFT pure component parameters:

$$
\begin{array}{rl}
f^{\mathrm{opt}}(y)=\min _{x} & f(x, y) \\
\text { s.t. } & g_{1}(x, y) \leq 0 \\
g_{2}(x, y)=0 \\
h(x, y)=0 \\
x_{\min } \leq x \leq x_{\max } \in \mathbb{R}^{\mathrm{m}} .
\end{array}
$$

The estimated objective function value $\hat{f}(y)$ is used as assessment criterion in the structure-mapping.

In this work, a CAMD algorithm is employed to design optimal molecules. This CAMD algorithm optimizes the molecular structure with respect to the performance estimate $\hat{f}(y)(2)$. In order to evaluate $\hat{f}(y)$, the PC-SAFT pure component parameters have to be known for each molecule. Lampe et al. (2015) employ a Group Contribution (GC) method in order to calculate PCSAFT pure component parameters from molecule structures. They use the homosegmented approach from Sauer et al. (2014) which they call GPC-SAFT. We use the same method as Lampe et al. (2015) and all nonpolar, non-associating groups they considered.

Following Lampe et al. (2015), the CAMD problem is formulated as mixed integer quadratic problem (MIQP) by employing the second-order Taylor approximation (2) as objective function:

$$
\begin{array}{ll}
\min _{n} \hat{f}(y) & \\
\text { s.t. } & G C(n)=y \\
& F E A S(n) \leq 0 \\
& A y \leq b_{\text {rel }} \\
& n \in \mathbb{Z}^{0}
\end{array}
$$

In this formulation, a fluid is described by a vector $n$ representing the functional groups constituting the molecular structure. The set of PC-SAFT pure component parameters $y$ are calculated with a GC method as described above $(G C(n)=y)$. A set of constraints $(F E A S(n) \leq 0)$ ensures feasible connectivity of the designed molecules (Struebing, 2011; Struebing et al., 2011). A convex hull $\left(A y \leq b_{\text {rel }}\right)$ as described in Section 2.1 is also used in the CAMD optimization. In order to permit the design of novel fluids, the convex hull is relaxed compared to the one used in the CoMT step, i.e. $b_{\text {rel }} \geq b$ (Lampe et al., 2015). The result of the MIQP is the optimal molecular structure of a real fluid.

A ranking of fluid candidates can be obtained by repeating the CAMD optimization with integer cuts (see e.g. Fazlollahi et al., 2012) which exclude previously found molecular structures from the design space.

\subsection{Final Process Optimizations}

The result of the structure-mapping step of CoMTCAMD is a ranking of candidate molecules. Since the objective function used for the structure-mapping is a second-order Taylor-approximation (2) as described in Section 2.3, process optimizations are performed with the identified real molecules in a final step in order to obtain the respective optimal process conditions and actual objective function values. These objective function values are also used to refine the ranking of the molecules.

\section{CoMT-CAMD with Modelica Mod- els}

As explained in Section 1, the integrated process and fluid design with the CoMT-CAMD approach was based on process models implemented in a procedural programming language so far. The contribution of this work is to enable the utilization of CoMT-CAMD based on object-oriented process modeling with Modelica. The utilization of this language facilitates the convenient development, adaption and reusability of models. In this way, the design of complex processes is enabled. Furthermore, equation-based modeling with Modelica is suited for the investigation of dynamic processes. The implementation of all steps of CoMT-CAMD based on Modelica is described in the subsequent sections. 


\subsection{PC-SAFT Modelica-Package}

Since the CoMT-CAMD approach is based on fluid property calculations with PC-SAFT, these calculations have to be available in Modelica to model the processes. Examples of such property calculations are the calculation of saturation pressures and temperatures as well as of caloric properties like specific enthalpies.

For this purpose, a PC-SAFT implementation written in the procedural language FORTRAN 90 is used for external property calculations. The interface with Modelica is constructed using the Modelica external function interface (Modelica Association, 2012).

According to Modelica Association (2012), however, interfaces are only supported with external FORTRAN 77 code. For this reason, FORTRAN 77 subroutines are implemented as wrappers for all relevant top-level subroutines in the FORTRAN 90 code. The wrapper subroutines are called by Modelica external functions (for details see Modelica Association (2012)). As the properties are calculated on mole basis in the external code and calculations on mass basis are desired for the process simulations in Modelica, additional functions for conversions between mole and mass basis as well as unit conversions are implemented.

In order to enable the convenient use of the PC-SAFT property calculations in Modelica, a Modelica package with the following functions is developed:

- Modelica external functions needed to call external subroutines.

- Additional functions for conversion between mole and mass basis as well as unit conversions.

- "Top-level" functions for each type of property calculation which call the external functions and functions for necessary conversions, perform any additional calculations required and return the desired thermodynamic properties.

As the focus of this work is embedding Modelica in the existing CoMT-CAMD framework, the PC-SAFT package is created independent of other libraries. The following types of property calculations are available in the package:

- Vapor-Liquid Equilibrium (VLE) calculations for pure components with specified saturation pressure or temperature.

- pT-flash calculations for mixtures.

- Bubble point and dew point calculations for mixtures.

- Property calculations for pure components and mixtures in subcooled liquid and superheated vapor states.

- Estimation of the critical point.

All these functions can be conveniently used in Modelica process models.

\subsection{CoMT-CAMD Based on Modelica Mod- els and GenOpt}

In order to facilitate the integrated optimization of processes and fluids based on object-oriented modeling, the process and all its equipment models are implemented in Modelica. For any required property calculation, the functions in the PC-SAFT package described in Section 3.1 are utilized.

In order to find the hypothetical optimum in the CoMT step, an objective function is defined. Then, the Modelica process model is used to search for the point in the solution space of process conditions and PCSAFT pure component parameters with the maximal objective function value.

One tool suited for this search is the open-source optimization program GenOpt (Generic Optimization Program) (Wetter, 2000; 2016) which provides algorithms for parametric runs as well as several optimization algorithms and can be coupled with Modelica models (Wetter, 2009).

In this work, parametric runs with GenOpt are used in the CoMT step to find the hypothetical optimal fluid and the corresponding optimal process conditions. The derivatives required for the Taylor-approximation of the objective function are approximated by finite differences. These are computed with MatLab from the results of the GenOpt calculations. As the output files of GenOpt are conveniently imported by MatLab, no special interface is required here.

The CAMD formulation described in Section 2.3 was implemented in the high-level modeling system GAMS (General Algebraic Modeling System) (Rosenthal, 2016) which is used to solve the MIQP with integer cuts to obtain a ranking of real fluids.

For the final process optimizations described in Section 2.4 parametric runs with GenOpt are used to find optimal process conditions. The workflow of the Modelica-based CoMT-CAMD is shown in Figure 2.

\section{Case Study: Design of a Geother- mal ORC Application}

The proposed molecular and process design with the CoMT-CAMD approach based on process modeling with Modelica is applied to a case study of a geothermal application for an Organic Rankine Cycle. Organic Rankine Cycles (ORCs) are used to transform low temperature heat into electric power (Colonna et al., 2015). The performance of ORCs depends strongly on the properties of the chosen working fluid. Therefore, the ORC is a very relevant case study for the integrated fluid and process design (Linke et al., 2015; Bao and Zhao, 2013). In the subsequent sections, the ORC process itself as well as the process model implemented in Modelica and the specifications of the case study are described. In Section 4.4, the results of the integrated fluid and process design are presented. 


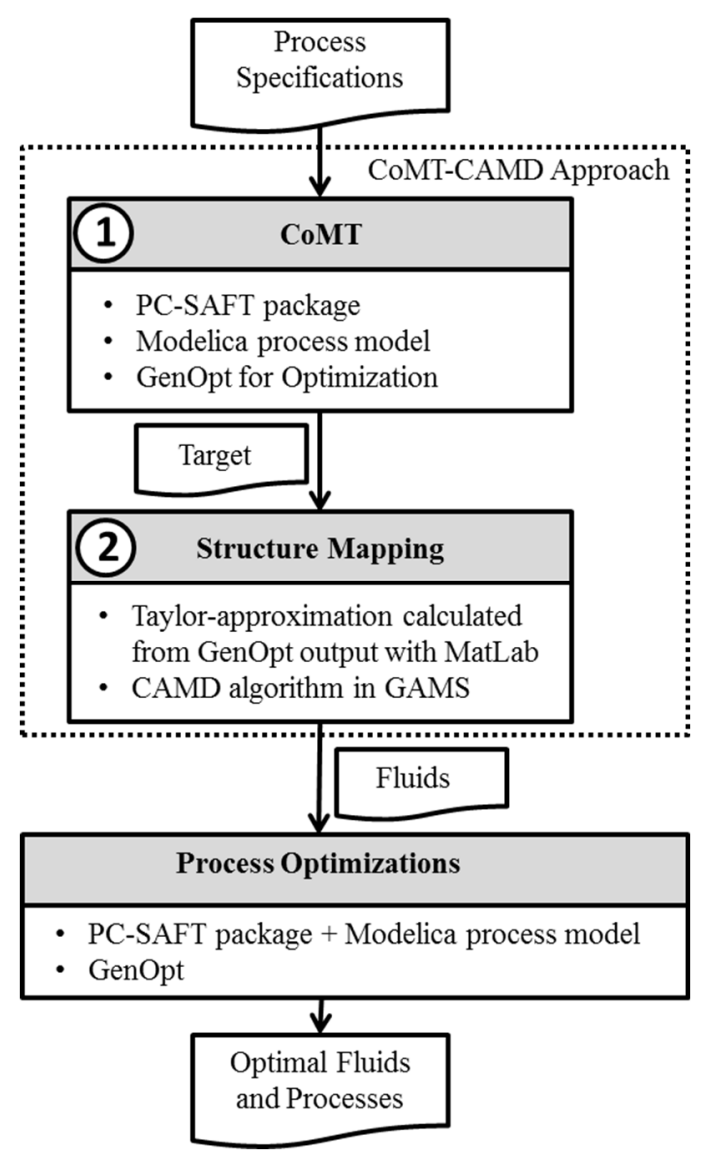

Figure 2: Workflow of the integrated molecular and process design with CoMT-CAMD including the tools used in the individual steps.

\subsection{Organic Rankine Cycles}

Organic Rankine Cycles (ORCs) are energy conversion processes with a sequence of process steps equivalent to the classical thermodynamic Rankine cycle (Colonna et al., 2015). The flowsheet and the temperature-entropy diagram of a basic ORC process are shown in Figure 3. In contrast to classical Rankine cycles which use water as working fluid, organic fluids are utilized in ORCs. The organic fluid can be tailored to specific applications, in particular the utilization of low temperature heat and cases with low power output where water becomes unfavorable (Colonna et al., 2015).

In the ORC process, the liquid working fluid (state 1 in Figure 3$)$ is pressurized in a pump $(1 \rightarrow 2)$ with power input $P_{P}$ before the vaporization in an evaporator $(2 \rightarrow$ 3) using heat $\left(\dot{Q}_{\text {evap }}\right)$ from the available heat source with inlet temperature $T_{\mathrm{HS} \text {,in. }}$. At the outlet of the evaporator (State 3 in Figure 3), the working fluid can be in a saturated or superheated vapor state. The vapor is expanded in a turbine $(3 \rightarrow 4)$ to gain the desired power output $P_{\mathrm{T}}$. Subsequently, the fluid is completely condensed in a condenser $(4 \rightarrow 1)$ by transferring heat $\left(\dot{Q}_{\text {cond }}\right)$ to a cooling medium with inlet temperature $T_{\mathrm{CW}}$,in in order to close the cycle.
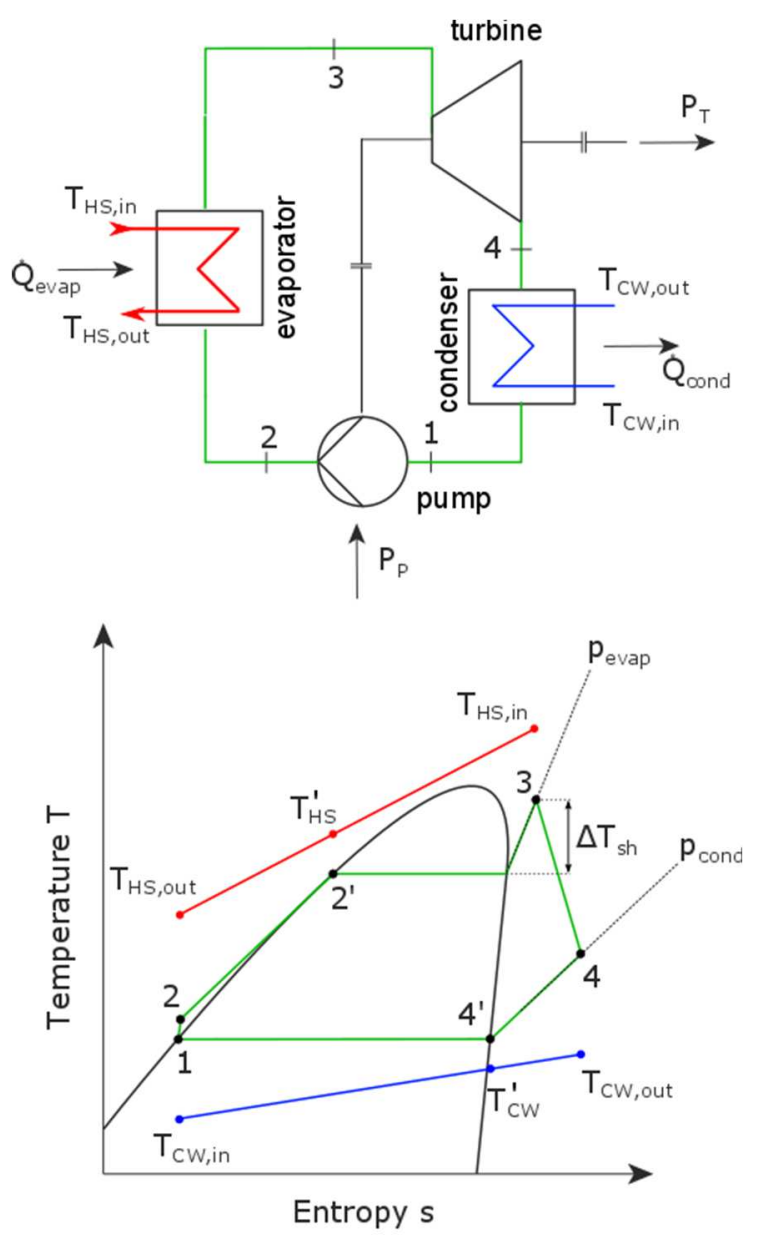

- heat source $(\mathrm{HS}) \quad$ cooling water $(\mathrm{CW})$

\section{_ working fluid}

Figure 3: Upper part: Flowsheet of a basic ORC process. Lower part: T-s diagram for ORC process, heat source and heat sink.

\subsection{Modelica ORC Model}

To demonstrate the integrated design of working fluid and process, the ORC is modeled assuming steady state conditions. Four equipment models are implemented and connected to a process model (cf. Figure 3): a pump, a turbine and two heat exchangers, namely the evaporator and the condenser. These equipment models are connected as shown in Figure 3 via suitable connectors to assemble an ORC process model. Although the use of libraries for modeling the ORC would be possible, no existing libraries are used in this work to keep the equipment models and connectors simple. The most important model equations and assumptions are presented in the following.

\subsubsection{Pump}

The pump is adiabatic and modeled based on an isentropic pressure increase and an isentropic efficiency $\eta_{\text {is,P: }}$ 


$$
\begin{aligned}
s_{\mathrm{P}, \text { in }} & =s_{\mathrm{P}, \text { out }}^{*} \\
h_{\mathrm{P}, \text { out }}^{*} & =f\left(p_{\text {evap }}, s_{\mathrm{P}, \text { out }}^{*}\right) \\
h_{\mathrm{P}, \text { out }} & =h_{\mathrm{P}, \text { in }}+\frac{h_{\mathrm{P}, \text { out }}-h_{\mathrm{P}, \text { in }}}{\eta_{\mathrm{is}, \mathrm{P}}}
\end{aligned}
$$

Here, $h_{\mathrm{P}, \text { in }}=h_{1}$ and $h_{\mathrm{P} \text {,out }}=h_{2}$ are the specific enthalpies at pump inlet and outlet, respectively, and $h_{\mathrm{P}, \text { out }}^{*}$ is the specific enthalpy at the pump outlet in case of isentropic pressure increase. $s_{\mathrm{P} \text {,in }}$ and $s_{\mathrm{P} \text {,out }}^{*}$ are the specific entropies at inlet and outlet conditions for the isentropic case. The pressure level of the evaporation is termed $p_{\text {evap }}$.

From the energy balance, the required power input can be obtained:

$$
P_{\mathrm{P}}=\dot{m}_{\mathrm{WF}}\left(h_{\mathrm{P}, \text { out }}-h_{\mathrm{P}, \text { in }}\right)
$$

where $\dot{m}_{\mathrm{WF}}$ is the working fluid mass flow rate and $P_{\mathrm{P}}$ the required power input.

\subsubsection{Turbine}

The adiabatic turbine is modeled in the same way as the pump based on an isentropic efficiency $\eta_{\mathrm{is}, \mathrm{T}}$ :

$$
\begin{aligned}
s_{\mathrm{T}, \text { in }} & =s_{\mathrm{T}, \text { out }}^{*} \\
h_{\mathrm{T}, \text { out }}^{*} & =f\left(p_{\text {cond }}, s_{\mathrm{T}, \text { out }}^{*}\right) \\
h_{\mathrm{T}, \text { out }} & =h_{\mathrm{T}, \text { in }} \\
& \quad+\eta_{\text {is, } \mathrm{T}}\left(h_{\mathrm{T}, \text { out }}^{*}-h_{\mathrm{T}, \text { in }}\right)
\end{aligned}
$$

where $p_{\text {cond }}$ is the pressure level of condensation. The power output of the turbine can be obtained from an energy balance as:

$$
P_{\mathrm{T}}=\dot{m}_{\mathrm{WF}}\left(h_{\mathrm{T}, \text { out }}-h_{\mathrm{T}, \text { in }}\right)
$$

\subsubsection{Evaporator}

It is assumed that pressure losses in the evaporator can be neglected such that preheating and evaporation take place on the same constant pressure level $p_{\text {evap. }}$ The geothermal heat source is assumed to be hot liquid water with a known inlet temperature $T_{\mathrm{HS}, \text { in }}$, mass flow $\dot{m}_{\mathrm{HS}}$ and constant specific heat capacity $c_{\mathrm{HS}}$.

The heat transferred is calculated from energy balances of the evaporator:

$$
\begin{aligned}
\dot{Q}_{\text {evap }} & =\dot{m}_{\mathrm{HS}} c_{\mathrm{HS}}\left(T_{\mathrm{HS}, \text { in }}-T_{\mathrm{HS}, \mathrm{out}}\right) \\
& =\dot{m}_{\mathrm{WF}}\left(h_{\text {evap,out }}-h_{\text {evap,in }}\right)
\end{aligned}
$$

where $T_{\mathrm{HS} \text {,out }}$ denotes the outlet temperature of the heat source and $h_{\text {evap,in }}$ and $h_{\text {evap,out }}$ the specific enthalpies of the working fluid at the evaporator inlet and outlet, respectively.

The specific enthalpy of the working fluid at the outlet of the evaporator $h_{\text {evap,out }}$ is calculated from the evaporator pressure $p_{\text {evap }}$ and outlet temperature $T_{3}$. The temperature $T_{3}$ is calculated from the saturation temperature of the working fluid and a degree of superheating $\Delta T_{\text {sh }}$.

\subsubsection{Condenser}

The condenser is modeled based on energy balances equivalent to those shown for the evaporator. It is assumed that cooling water with known inlet temperature $T_{\mathrm{CW} \text {,in }}$ and a known constant specific heat capacity $c_{\mathrm{CW}}$ is used as cooling medium. The required mass flow of the cooling water is calculated from a given temperature increase of $5 \mathrm{~K}$. The outlet state of the working fluid is assumed to be a saturated liquid state. Pressure losses in the condenser are neglected.

\begin{tabular}{|c|c|c|c|c|c|}
\hline Parameter & Symbol & Value & Parameter & Symbol & Value \\
\hline $\begin{array}{l}\text { heat source mass flow } \\
\text { rate }\end{array}$ & $\dot{m}_{\mathrm{HS}}$ & $66 \mathrm{~kg} / \mathrm{s}$ & $\begin{array}{l}\text { cooling water in- } \\
\text { let/outlet temperature }\end{array}$ & $T_{\mathrm{CW}, \text { in }} / T_{\mathrm{CW} \text {,out }}$ & $15^{\circ} \mathrm{C} / 20^{\circ} \mathrm{C}$ \\
\hline $\begin{array}{l}\text { heat source inlet tem- } \\
\text { perature }\end{array}$ & $T_{\mathrm{HS}, \text { in }}$ & $120^{\circ} \mathrm{C}$ & $\begin{array}{l}\text { cooling water heat ca- } \\
\text { pacity }\end{array}$ & $c_{\mathrm{CW}}$ & $4200 \mathrm{~J} /(\mathrm{kg} \mathrm{K})$ \\
\hline $\begin{array}{l}\text { heat source specific } \\
\text { heat capacity }\end{array}$ & $c_{\mathrm{HS}}$ & $4200 \mathrm{~J} /(\mathrm{kg} \mathrm{K})$ & $\begin{array}{l}\text { pinch temperature dif- } \\
\text { ference }\end{array}$ & $\Delta T^{\text {pinch }}$ & $5 \mathrm{~K}$ \\
\hline $\begin{array}{l}\text { min. and max. abso- } \\
\text { lute pressure }\end{array}$ & $p^{\min / \max }$ & $1 \mathrm{bar} / 50 \mathrm{bar}$ & $\begin{array}{l}\text { min. vapor fraction at } \\
\text { turbine outlet }\end{array}$ & $\beta^{\text {turb,min }}$ & 0.95 \\
\hline max. reduced pressure & $p^{r, \max }$ & 0.8 & & & \\
\hline
\end{tabular}

\subsection{Specifications of the Case Study and the Optimization Problem}

The specifications of the case study are based on the subcritical geothermal ORC application presented by Heberle and Brüggemann (2010) and are shown in Table 1 . The ORC designed in our work is medium-sized regarding its power capacity and utilizes low-temperature heat from a geothermal source with a maximum temperature of $120{ }^{\circ} \mathrm{C}$.

Table 1: Specifications of the case study based on Heberle and Brüggemann (2010). 
Table 2: Optimal process conditions and PC-SAFT parameters of the hypothetical optimal fluid resulting from the CoMT step as well as the achieved net power output. Values at their bounds are marked with a + .

\begin{tabular}{lcrlcr}
\hline Parameter & Symbol & Value & Parameter & Symbol & Value \\
\hline condensation pressure & $p_{\text {cond }}^{*}$ & $7.1 \mathrm{bar}$ & segment number & $m^{*}$ & 3.15 \\
evaporation pressure & $p_{\text {evap }}^{*}$ & $25.7 \mathrm{bar}$ & segment diameter & $\sigma^{*}$ & $3.45 \AA$ \\
degree of superheating & $\Delta T_{\mathrm{sh}}^{*}$ & $0^{\circ} \mathrm{C}$ & segment dispersion energy & $(\varepsilon / k)^{*}$ & $164.0 \mathrm{~K}$ \\
$\begin{array}{l}\text { working fluid mass flow } \\
\text { rate }\end{array}$ & $\dot{m}_{\mathrm{WF}}^{*}$ & $70.0 \mathrm{~kg} / \mathrm{s}$ & net power output & $P_{\text {net }}^{*}$ & $1.9 \mathrm{MW}$ \\
\hline
\end{tabular}

The degrees of freedom $x$ of the ORC process considered in the optimization are the pressure levels of evaporation $p_{\text {evap }}$ and condensation $p_{\text {cond }}$, the mass flow rate of the ORC working fluid $\dot{m}_{W F}$ and the degree of superheating at the evaporator outlet $\Delta T_{\text {sh }}$. The PCSAFT pure component parameters $y$ considered for the working fluid optimization are the segment number $m$, segment diameter $\sigma$ and segment dispersion energy $(\varepsilon / k)$ (cf. Section 2.2).

Bounds on the process degrees of freedom are minimal and maximal absolute pressures $p^{\text {min }}$ and $p^{\max }$ of 1 bar and 50 bar, respectively. Additionally, an upper bound of the reduced pressure $p^{\text {r,max }}$, defined as the absolute pressure $p$ divided by the critical pressure $p^{\mathrm{c}}$, of 0.8 is used because only subcritical ORCs are considered. In order to avoid damage of the turbine, it is important in practice to avoid the formation of liquid droplets during expansion. Thus, a minimal vapor fraction $\beta^{\text {turb,min }}$ of 0.95 at the turbine outlet is used as a further constraint (see Table 1).

Additional constraints arise to ensure that the temperature differences between the heat source and the working fluid in the evaporator and between the working fluid and the cooling water in the condenser do not violate the specified pinch temperature difference $\Delta T^{\text {pinch }}$ of $5 \mathrm{~K}$ at any point. As can be seen in the T-s diagram shown in Figure 3, possible pinch points in the evaporator are at the inlet and outlet and at the point where the working fluid reaches the saturation temperature. Possible pinch points in the condenser are at the condenser inlet and, in case the working fluid is superheated at the inlet, at the point where it first reaches a saturated state.

The net power output $P_{\text {net }}$ of the ORC is used as objective function for the optimization and can be calculated from the required power input of the pump $P_{\mathrm{P}}$ and the power output of the turbine $P_{\mathrm{T}}$ as

$$
f(x, y)=P_{\text {net }}=-\left(P_{\mathrm{T}}+P_{\mathrm{P}}\right)
$$

This definition of $P_{\text {net }}$ leads to a positive objective function $f(x, y)$ which is maximized in the optimization.

\subsection{Results}

To identify an optimal working fluid and the corresponding optimal process conditions for the considered
ORC the CoMT-CAMD approach is used (see Figures 1 and 2). First, an optimal hypothetical fluid and corresponding process conditions are obtained as target in the CoMT step. Subsequently, fluids are designed in the CAMD step which best match the target.

The results of the CoMT step are shown in Table 2. The PC-SAFT pure component parameters shown in the table correspond to a hypothetical optimal fluid. The net power output $P_{\text {net }}$ of the target is $1.9 \mathrm{MW}$. This target value serves as an upper bound as it represents the highest power output achievable for all hypothetical fluids represented by PC-SAFT in the considered convex hull (cf. Section 2.1).

In order to determine a ranking of real fluids which best match the hypothetical optimum, a ComputerAided Molecular Design (CAMD) algorithm is employed as described in Sections 2.3 and 3.2. Subsequently, the optimal process parameters and corresponding net power output $P_{\text {net }}$ for the individual fluids are obtained from process optimizations (cf. Sections 2.4 and 3.2).

The top 10 fluids of the CAMD step are presented in Table 3 together with the respective net power outputs resulting from the process optimizations.

As can be seen in Table 3, the top fluids suggested by the CAMD algorithm are all linear and branched alkanes and alkenes with a maximum of 6 carbon atoms. We identify propene as the best working fluid for the ORC with a net power output $P_{\text {net }}$ of $1.45 \mathrm{MW}$. This value is $24 \%$ less than the target value of the hypothetical molecule.

From Table 3, it can be seen that the net power outputs determined in the process optimizations do not perfectly match the ranking from the CAMD step. The objective function used in the CAMD step is a Taylor-approximation of the real objective function of the integrated optimization problem as explained in Section 2. As the Taylor-approximation does not match the real objective function perfectly, the ranking from the CAMD step slightly deviates from the final ranking based on optimized net power outputs. It is therefore recommended to generate a list of candidates and not only one working fluid. This behavior was also found in earlier implementations of the CoMT-CAMD approach (cf. Lampe et al., 2014; 2015) and is thus not specific for the Modelica-based implementation. 
Table 3: Top fluids resulting from the CAMD step and corresponding net power output determined using process optimizations. The order is according to the ranking of the CAMD step. The sorted rank according to the results of the individual process optimizations is shown in parentheses.

\begin{tabular}{lcc}
\hline $\begin{array}{l}\text { Rank } \\
\text { (Sorted Rank) }\end{array}$ & Fluid & Net Power Output \\
\hline $1(2)$ & Propane & $1.44 \mathrm{MW}$ \\
$2(6)$ & Neopentane & $1.31 \mathrm{MW}$ \\
$3(1)$ & Propene & $1.45 \mathrm{MW}$ \\
$4(9)$ & Propyne & $1.24 \mathrm{MW}$ \\
$5(3)$ & Isobutene & $1.38 \mathrm{MW}$ \\
$6(4)$ & 2-Butene & $1.38 \mathrm{MW}$ \\
$7(7)$ & Butane & $1.25 \mathrm{MW}$ \\
$8(5)$ & $1-B u t e n e$ & $1.33 \mathrm{MW}$ \\
$9(10)$ & Neohexane & $1.11 \mathrm{MW}$ \\
$10(8)$ & 1-Butyne & $1.25 \mathrm{MW}$ \\
\hline
\end{tabular}

Clearly, the net power output of the target given in Table 2 serves as an upper bound of the objective function. Therefore, the target is not reached by the designed real working fluids.

In order to find the target in the CoMT step with GenOpt, about $2 \cdot 10^{5}$ evaluations of the process model are required. On the other hand, an individual process optimization for one working fluid requires about $10^{4}$ evaluations of the process model. Thus, the effort for the integrated molecular and process design with the Modelica-based CoMT-CAMD is similar to the effort for process optimizations for about 30 working fluid candidates. This shows the strength of CoMT-CAMD, where thousands of different molecules are considered in the structure-mapping. Additionally, there are further improvements possible compared to the current implementation. As presented in Section 3.2, parametric runs with GenOpt are used in the CoMT step to find the optimal hypothetical fluid and the corresponding optimal process conditions. Employing gradient-based optimization instead of the parametric runs has the potential to further decrease the computational effort while substantially increasing the accuracy.

\section{Conclusion}

In this work, we propose the Continuous-Molecular Targeting - Computer-Aided Molecular Design (CoMTCAMD) approach based on object-oriented process modeling with Modelica. The CoMT-CAMD approach originally proposed by Bardow et al. (2010) enables the simultaneous optimization of processes and fluids without any fluid pre-selection by utilizing the physicallybased equation of state PC-SAFT. So far, implementations of the CoMT-CAMD design approach were based on process modeling utilizing procedural programming languages. Thus, the reusability of models has been hindered and the investigation of process variants as well as complex processes has been cumbersome. For this reason, it is shown in this work how integrated process and molecular design with CoMT-CAMD can be based on object-oriented process modeling with Modelica. The proposed implementation uses the Modelica external function interface for external property calculations with PC-SAFT and the optimization tool GenOpt for the search of optimal hypothetical fluids and process conditions. Additionally, a Computer-Aided Molecular Design algorithm implemented in GAMS is used in order to design real fluids which best match the hypothetical optimum. The Modelica-based CoMT-CAMD implementation is demonstrated for the design of a subcritical geothermal Organic Rankine Cycle (ORC). The results show that the approach efficiently identifies optimal ORC processes and working fluids. The CoMT-CAMD approach implemented in an object-oriented language for process modeling allows for convenient and efficient integrated process and fluid design for complex processes. Future work will address the use of deterministic optimization and the utilization of libraries for the Modelica-based CoMT-CAMD.

\section{Acknowledgements}

We thank the Deutsche Forschungsgemeinschaft (DFG) for funding this work (BA2884/4-1). Furthermore, the authors thank Heike Schreiber, Matthias Lampe and Christian Schulze for valuable discussions.

\section{References}

Claire S. Adjiman, Amparo Galindo and George Jackson. Molecules Matter: the Expanding Envelope of Process Design. Computer Aided Chemical Engineering, 34:55-64, 2014. doi: 10.1016/B978-0-444-63433-7.50007-9.

Junjiang Bao and Li Zhao. A Review of Working Fluid and Expander Selections for Organic Rankine Cycle. Renewable and Sustainable Energy Reviews, 24:325-342, 2013. doi: 10.1016/j.rser.2013.03.040.

André Bardow, Klaas Steur and Joachim Gross. ContinuousMolecular Targeting for Integrated Solvent and Process Design. Industrial \& Engineering Chemistry Research, 49(6):2834-2840, 2010. doi: 10.1021/ie901281w.

Jakob Burger, Vasileios Papaioannou, Smitha Gopinath, George Jackson, Amparo Galindo and Claire S. Adjiman. A Hierarchical Mmethod to Integrated Solvent and Process Design of Physical $\mathrm{CO}_{2}$ Absorption Using the SAFT- $\gamma$ Mie Approach. AIChE Journal, 61(10):3249-3269, 2015. doi: 10.1002/aic.14838.

Piero Colonna, Emiliano Casati, Carsten Trapp, Tiemo Mathijssen, Jaakko Larjola, Teemu Turunen-Saaresti and Antti Uusitalo. Organic Rankine Cycle Power Systems. Journal of Engineering for Gas Turbines and Power, 137(10):100801, 2015. doi: 10.1115/1.4029884.

Samira Fazlollahi, Pierre Mandel, Gwenaelle Becker and Francois Maréchal. Methods for Multi-Objective Investment and Operating Optimization of Complex Energy Systems. Energy, 45(1):12-22, 2012. doi: 10.1016/j.energy.2012.02.046. 
Peter Fritzson. Modelica - A Language for Equation-Based Physical Modeling and High Performance Simulation. Applied Parallel Computing, 1541:149-160, 1998. ISSN: 0302-9743.

Rafiqul Gani. Chemical Product Design. Computers \& Chemical Engineering, 28(12):2441-2457, 2004. doi: 10.1016/j.compchemeng.2004.08.010.

Smitha Gopinath, George Jackson, Amparo Galindo and Claire S. Adjiman. Outer approximation algorithm with physical domain reduction for computer-aided molecular and separation process design. AIChE Journal, 62(9):34843504, 2016. doi: 10.1002/aic.15411.

Joachim Gross and Gabriele Sadowski. Perturbed-Chain SAFT. Industrial \& Engineering Chemistry Research, 40(4):1244-1260, 2001. doi: 10.1021/ie0003887.

Joachim Gross and Gabriele Sadowski. Application of the Perturbed-Chain SAFT Equation of State to Associating Systems. Industrial \& Engineering Chemistry Research, 41(22):5510-5515, 2002. doi: 10.1021/ie010954d.

Joachim Gross. An Equation-of-State Contribution for Polar Components. AIChE Journal, 51(9):2556-2568, 2005. doi: 10.1002/aic.10502.

Joachim Gross and Jadran Vrabec. An Equation-of-State Contribution for Polar Components. AIChE Journal, 52(3):1194-1204, 2006. doi: 10.1002/aic.10683.

Florian Heberle and Dieter Brüggemann. Exergy Based Fluid Selection for a Geothermal Organic Rankine Cycle for Combined Heat and Power Generation. Applied Thermal Engineering, 30(11-12):1326-1332, 2010. doi: 10.1016/j.applthermaleng.2010.02.012.

Matthias Lampe, Marina Stavrou, Hanns M. Bücker, J. Gross and A. Bardow. Simultaneous Optimization of Working Fluid and Process for Organic Rankine Cycles Using PCSAFT. Industrial \& Engineering Chemistry Research, 53(21):8821-8830, 2014. doi: 10.1021/ie5006542.

Matthias Lampe, Marina Stavrou, Johannes Schilling, Elmar Sauer, Joachim Gross and André Bardow. Computer-Aided Molecular Design in the Continuous-Molecular Targeting Framework Using Group-Contribution PC-SAFT. Computers \& Chemical Engineering, 81:278-287, 2015. doi: 10.1016/j.compchemeng.2015.04.008.

Patrick Linke, Athanasios Papadopoulos and Panos Seferlis. Systematic Methods for Working Fluid Selection and the Design, Integration and Control of Organic Rankine Cycles-A Review. Energies, 8(6):4755-4801, 2015. doi: 10.3390/en8064755.

Modelica Association. Modelica - A Unified Object-Oriented Language for Systems Modeling - Language Specification Version 3.3. URL $=$ " https://www. modelica. org/documents/ModelicaSpec33. Pdf", 2012.

Athanasios I. Papadopoulos and Patrick Linke. Integrated Solvent and Process Selection for Separation and Reactive Separation Systems. Chemical Engineering and Processing: Process Intensification, 48(5):1047-1060, 2009. doi: 10.1016/j.cep.2009.02.004.

Frances E. Pereira, Emmanuel Keskes, Amparo Galindo, George Jackson and Claire S. Adjiman. Integrated Design of $\mathrm{CO}_{2}$ Capture Processes from Natural Gas. In: Efstratios Pistikopoulos, Michael Georgiadis and Eustathios S. Kik- kinides. Editors. Process Systems Engineering: Energy Systems Engineering. Weinheim: Wiley-VCH Verlag GmbH \& Co. KG, pp. 231-248, 2008.

Frances E. Pereira, Emmanuel Keskes, Amparo Galindo, George Jackson and Claire S. Adjiman. Integrated Solvent and Process Design Using a SAFT-VR Thermodynamic Description. Computers \& Chemical Engineering, 35(3):474-491, 2011. doi: 10.1016/j.compchemeng.2010.06.016.

Richard E. Rosenthal. GAMS - a User's Guide, GAMS Release 24.6.1. URL: http://www.gams.com/help/topic/ gams.doc/userguides/GAMSUsersGuide.pdf. 2016.

Dennis Roskosch and Burak Atakan. Reverse Engineering of Fluid Selection for Thermodynamic Cycles with Cubic Equations of State, Using a Compression Heat Pump as Example. Energy, 81:202-212, 2015. doi: 10.1016/j.energy.2014.12.025.

Nikolaos V. Sahinidis, Mohit Tawarmalani and Minrui Yu. Design of Alternative Refrigerants via Global Optimization. AIChE Journal, 49(7):1761-1775, 2003. doi: 10.1002/aic.690490714.

Elmar Sauer, Marina Stavrou and Joachim Gross. Comparison between a Homo- and a Heterosegmented Group Contribution Approach Based on the Perturbed-Chain Polar Statistical Associating Fluid Theory Equation of State. Industrial \& Engineering Chemistry Research, 53(38):14854-14864, 2014. doi: 10.1021/ie502203w.

Marina Stavrou, Matthias Lampe, André Bardow and Joachim Gross. Continuous Molecular Targeting-Computer-Aided Molecular Design (CoMT-CAMD) for Simultaneous Process and Solvent Design for $\mathrm{CO}_{2}$ Capture. Industrial \& Engineering Chemistry Research, 53(46):18029-18041, 2014. doi: 10.1021/ie502924h.

Heiko Struebing. Identifying Optimal Solvents for Reactions Using Quantum Mechanics and Computer-Aided Molecular Design. Ph.D. thesis, Imperial CollegeLondon, London, 2011.

Heiko Struebing, Amparo Galindo and Claire S. Adjiman. Optimal Solvent Design for Reactions Using Computer-Aided Molecular Design. URL: http://www.minlp.org/library/problem $/ \mathrm{mod} /$ index.php ?lib=MINLP\&i=180\&pi=137. 2011.

Michael Wetter. Design Optimization with GenOpt. Building Energy Simulation, (21):19-28, 2000.

Michael Wetter. Modelica-Based Modelling and Simulation to Support Research and Development in Building Energy and Control Systems. Journal of Building Performance Simulation, 2(2):143-161, 2009. doi: $10.1080 / 19401490902818259$.

Michael Wetter. GenOpt - Generic Optimization Program User Manual - Version 3.1.1. URL: https:// http://simulationresearch.lbl.gov/GO/download/manual-3-1-1.pdf, 2016. 